Revista de Investigación Educativa 27

julio-diciembre, 2018 | ISSN 1870-5308 | Xalapa, Veracruz

Instituto de Investigaciones en Educación | Universidad Veracruzana

\title{
Factores personales-institucionales que impactan el rendimiento académico en un posgrado en educación
}

\section{Personal and institutional factors affecting academic performance in a postgraduate program in education}

\author{
Mario José Martín Pavón ${ }^{a}$ \\ Dora Esperanza Sevilla Santo ${ }^{b}$ \\ Cristina Jenaro Río ${ }^{b}$
}

Recibido: 19 de junio de 2017 Aceptado: 09 enero de 2018

La presente investigación tuvo como objetivo identificar los factores personales e institucionales que determinan el rendimiento académico de los estudiantes de la Maestría en Investigación Educativa, ofertada por la Facultad de Educación de la Universidad Autónoma de Yucatán, México. El estudio se desarrolló bajo el paradigma cuantitativo. Los participantes pertenecen a las cohortes 2004-2014, y fueron clasificados en estudiantes de alto o bajo rendimiento. La información se recolectó con un cuestionario estructurado que indagó sobre: conocimientos previos, antecedentes académicos, habilidad para trabajar en equipo, expectativas del programa, motivación, autoestima, bienestar psicológico, procesos académicosadministrativos y desempeño del asesor y tutor. El análisis de la información incluyó procesos comparativos, estudio de relaciones, análisis de regresión y análisis discriminante. El estudio reportó que la experiencia en investigación, los hábitos de estudio y la autoestima son los factores que más influyen en el rendimiento académico de los estudiantes del programa.

Palabras clave: Desempeño académico; estudiantes de posgrado; características del estudiante; contexto escolar.

a Doctor, Profesor de carrera titular B, Universidad Autónoma de Yucatán, México.

凶mario.martin@correo.uady.mx.

b Doctora, Profesor-Investigador titular C, Universidad Autónoma de Yucatán, México.

凶dora.sevilla@correo.uady.mx.

'Doctora, Profesor-Investigador, Universidad de Salamanca, España.

凶 crisje@usal.es. 
The present research aims to identify the personal and institutional factors that determine the academic performance Master in Educational Research students, offered by the Faculty of Education of the Autonomous University of Yucatan, Mexico. The study was developed under the quantitative paradigm. Participants belong to the 2004-2014 cohorts, and were classified as high or low performing students. The information was collected with a structured questionnaire that inquired about: previous knowledge, academic background, team-work skills, program expectations, motivation, self-esteem, psychological well-being, academicadministrative processes and consultant and tutor performance. The analysis of the information included comparative processes, relationship study, and regression and discriminant analyses. The study reported that research experience, study habits, and self-esteem were the most influential factors in the academic performance of students in the program.

Keywords: Academic performance; postgraduate students; student characteristics; school context.

\title{
Factores personales-institucionales que impactan el rendimiento académico en un posgrado en educación
}

\author{
Personal and institutional factors affecting academic \\ performance in a postgraduate program in education
}

\section{Antecedentes}

$\mathrm{H}^{\circ}$ oy en día, la ciudadanía confiere a la educación la responsabilidad de lograr un mundo más equitativo y eficiente. Esta exigencia se presenta con mayor fuerza en el nivel de posgrado; de aquí que exista un creciente interés de las instituciones educativas por encontrar mecanismos que promuevan el rendimiento académico de sus alumnos, para lo cual se han dado a la tarea de indagar sobre los factores que influyen en el desempeño, rezago y deserción (Rivera, 2011). 
Es así que existen estudios que han tratado de identificar los factores que rodean al estudiante y que inciden de manera positiva o negativa en su rendimiento académico, los cuales podrían agruparse en dos categorías principales: aquellos que reportan la importancia de aspectos de índole social y los que se vinculan con el análisis de aspectos que atañen al centro educativo (Muñoz, Martínez \& Armengol, 2010). Estos últimos, junto con los factores personales, son los que serán abordados en el presente artículo, dada la factibilidad de ser atendidos por las instituciones educativas.

Un aspecto que obstaculiza el esfuerzo de las Instituciones de Educación Superior (IES) por mejorar el rendimiento académico de sus estudiantes, se vincula con la diversidad de necesidades de los diferentes actores involucrados en los procesos formativos, así como por el contexto en el que dichos procesos se gestan (Albornoz, 2004). En este sentido y a manera de ejemplo, Martín (2015) menciona que la visión de considerar los entornos en el estudio del rendimiento académico surge de la teoría ecosistémica del desarrollo humano de Bronfenbrenner (1987), la cual afirma que los estudiantes se ven afectados por los cambios en los entornos inmediatos en los que viven, así como por las relaciones que se presentan entre éstos y los contextos en que se incluyen.

A partir de esta idea, Rivera (2011, como se citó en Martín, 2015) concluye que los ecosistemas definidos por Brofenbrenner son determinantes del rendimiento académico de los estudiantes e identifica en su estudio diversos factores como: el rol de los maestros, los programas de estudio y los factores ambientales del salón (microsiste$\mathrm{ma}$ ); la seriación de las materias, el entorno familiar y la profesión u oficio de los padres (mesosistema); el costo de la carrera, el lugar de estudio, los problemas de salud y la situación laboral (exosistema); la movilidad urbana, así como el medio ambiente socioeconómico y político que rodea al alumno (macrosistema).

Como puede verse, existe un amplio espectro de factores vinculados al ambiente; no obstante, de acuerdo con Alexander (2006), son la cultura, la tecnología y las prácticas instructivas los que más influyen en el rendimiento académico de los estudiantes. Este interés por considerar los factores del ambiente para la mejora de los procesos formativos no resulta exclusivo de las IES; también existen otras instancias, como la Organización de Estados Iberoamericanos (OEI), que asumen el carácter multifactorial de este constructo destacando las dimensiones social, económica y política.

Entre los aspectos del ambiente que se vinculan con las instituciones educativas, la literatura (Méndez, 2011; Paredes \& Paredes, 2009) refiere, entre otros: la gestión de la organización, las políticas educativas, las estrategias y cargas docentes, los 
alumnos por grupo, así como los criterios de evaluación. De manera particular, un supuesto importante en estos estudios refiere que "las expectativas del estudiante hacia la institución juegan un papel importante en su desempeño académico" (Torres \& Rodríguez, 2006, como se citó en Martín, 2015, p. 19). Todos los estudios señalados evidencian que estudiar el rendimiento académico y los factores que lo determinan es un campo muy complejo, dado que existe un número significativo de aspectos que no dependen directamente del sujeto que aprende, pero que será necesario considerar al momento de establecer estrategias al interior de las instituciones educativas para lograr el avance en el proceso educativo de todos los alumnos.

Por otra parte, existe un importante número de estudios que buscan explicar la influencia de los factores personales en el rendimiento académico. En relación con este tipo de aspectos, hay investigadores que afirman que el alumno es el único responsable de su proceso de aprendizaje y que de él dependerá el éxito que alcance (Cruz, 2006). En este sentido, la literatura reporta diversos factores vinculados con el estudiante, distinguiendo entre no cognitivos (motivación, intereses, expectativas, autorregulación, entre otros) y cognitivos (conocimientos específicos, hábitos de estudio, antecedentes académicos, etcétera). Al respecto, Buchmann y Diprete (2006) identifican como determinantes del rendimiento académico a la motivación, las emociones, los estilos de aprendizaje, los hábitos de estudio y la importancia que el estudiante confiere a las tareas académicas.

Como puede verse, la preocupación de las ies por identificar los factores que impactan el rendimiento académico de los estudiantes no es un tema nuevo; sin embargo, permanece vigente dados los elevados índices de deserción y rezago que se presentan en los programas, lo cual genera dudas sobre la forma en que estos se implementan y sobre la calidad de los servicios que las instituciones ofrecen y que soportan los planes de estudio.

\section{Planteamiento del problema}

A nivel internacional, una de las problemáticas que más preocupa a las autoridades educativas es la deserción y el rezago escolar. De manera específica, en el nivel superior en España, la deserción se encuentra alrededor del 26\% (López-Justicia, Hernández, Fernández, Sánchez \& Chacón, 2008) y para el caso de México, en el posgrado (nivel que nos ocupa), esta cifra es cercana a 20\% (Rivera, 2011). Además, los índices 
de eficiencia terminal siguen siendo bajos, en México, inferior a 12\% (Instituto Nacional de Estadística y Geografía [INEGI], 2005). Con relación al posgrado, en el ciclo escolar 2015-2016, el porcentaje de egreso fue de 33.7\% y el de graduación de 25\%; para el caso de Yucatán, el egreso fue de $36 \%$ y la graduación de $20 \%$ (ANUIES, 2017). Asimismo, en la universidad que oferta el programa en estudio estos porcentajes fueron de $27 \%$ y $13.4 \%$, respectivamente.

Por otra parte, de acuerdo con los registros del departamento de control escolar de la Facultad de Educación de la Universidad Autónoma de Yucatán, que oferta la Maestría en Investigación Educativa (MIE), el índice de eficiencia terminal es de 29.6\%; valor que compromete su acreditación y evidencia la baja capacidad predictiva del rendimiento académico de los indicadores de desempeño utilizados para la selección de aspirantes, y la existencia de otros factores que impactan el rendimiento académico no considerados.

Entre las causas de la deserción señaladas por diferentes autores (Armengol \& Castro, 2004; Núñez \& González-Pienda, 2006), se encuentran la falta de habilidad de los alumnos para comprender y analizar textos, así como la falta de capacidad para autogestionar su aprendizaje, lo que propicia que no puedan cumplir con las demandas de los programas en los que se encuentran matriculados, propiciando un bajo rendimiento académico y una disminución en la eficiencia terminal de los programas.

A este respecto, Espinosa (2016) menciona que existe relación causa-efecto entre el rendimiento académico y la eficiencia terminal de los programas, por lo que atender los factores que inciden en el primero puede ser una estrategia para mejorar esta última (Domínguez, Sandoval, Cruz \& Pulido, 2014; Guzmán, 2012; Ibarra \& Michalus, 2010). Además, el estudio del rendimiento académico y la eficiencia terminal no sólo tiene un impacto académico, sino político y social, ya que a partir de éste se puede promover el desarrollo de una educación inclusiva que fomente el avance de la sociedad.

En este contexto, de acuerdo con el reporte de la UNESCO de 2006, el abandono de los estudios universitarios tiene un alto costo para las instituciones educativas; para el caso particular de México, éste asciende a 415 millones de dólares, a lo que deben agregarse las repercusiones en el ámbito de la salud física y mental de los estudiantes que sufren dicha experiencia (Torres, Osuna \& Liekens, 2011). Otra implicación que tiene el fenómeno de la deserción se vincula con el hecho de que los organismos acreditadores consideran como un indicador de calidad de los programas a la eficiencia 
terminal, por lo que al no poder cumplir con este requerimiento se compromete su acreditación y la posibilidad de contar con un sistema de becas que beneficie a los estudiantes, sin las cuales muchos de ellos no podrían realizar sus estudios.

A partir de este panorama, se observa la necesidad de realizar investigaciones orientadas al estudio de los factores que determinan el rendimiento académico y, por ende, la eficiencia terminal de este tipo de programas. Aunque se ha investigado en diversos niveles educativos el estado de la eficiencia terminal, el rezago y la deserción, en el nivel de posgrado es limitado el trabajo realizado y existe un vacío de información. Esta situación llama la atención, sobre todo cuando se trata de programas que cuentan con un reconocimiento como el de CONACYT, donde uno de los indicadores de calidad es precisamente la eficiencia terminal. De aquí que se plantee la siguiente pregunta de investigación.

\subsection{Pregunta de investigación}

¿Cuáles son los factores que predicen el rendimiento académico de los estudiantes de la Maestría en Investigación Educativa?

\subsection{Objetivos}

1. Identificar los factores personales e institucionales que determinan el rendimiento académico de los estudiantes del programa en estudio.

2. Determinar el nivel de importancia de estos factores sobre el rendimiento académico de los estudiantes del programa en estudio.

3. Determinar la capacidad predictiva conjunta de estos factores sobre el rendimiento académico.

\section{Marco teórico}

El concepto de rendimiento académico ha sido definido de diferentes maneras, siendo compleja su definición dado su carácter multifactorial. En este sentido, Díaz (1995) comenta que dicha definición resulta importante en los estudios de tipo predictivo, ya que el uso de una sola medida del rendimiento como las calificaciones, esconde los efectos que sobre la formación del estudiante tienen los factores del contexto y otros 
que atañen al propio estudiante. Este autor afirma que la definición debe considerar los aprendizajes adquiridos para continuar los estudios y la actividad laboral; no obstante, muchas investigaciones adoptan una concepción simple en términos de las calificaciones o del promedio de éstas (Torres \& Merino, 2010).

Por otra parte, Ibarra y Michalus (2010) comentan que es común considerar el rendimiento como el cociente entre el número de materias aprobadas y el tiempo de permanencia en el sistema en el que se encuentra matriculado el estudiante.

En cuanto a los factores que impactan el rendimiento académico de los estudiantes, la literatura reporta que los maestros, los programas de estudio, el ambiente del salón de clases, la seriación de las materias, el entorno familiar, la profesión u oficio de los padres, el costo de la carrera, el lugar de estudio, los problemas de salud, la situación laboral, la movilidad urbana, la autoestima, así como el medio ambiente socioeconómico y político que rodea al alumno, condicionan su desempeño en el aula (Bain, Fedynich \& Knight, 2009; Rivera, 2011). Esta diversidad de factores que se reporta deja entrever que los condicionantes del rendimiento académico pueden ser clasificados en cuatro categorías: institucionales, pedagógicos, psicosociales y sociodemográficos (Montero, Villalobos \& Valverde, 2007).

Al respecto, Vargas, Aburto, Cortés, Álvarez, Ramírez, Farfán y Heinze (2010, como se citó en Martín, 2015) hacen referencia a:

factores personales (competencias cognitivas, motivación, autoeficacia percibida, bienestar psicológico, inteligencia, sexo, formación académica previa a la universidad y nota de acceso a ésta), sociales (diferencias sociales, entorno familiar, nivel educativo de los progenitores y variables sociodemográficas) e institucionales (metodologías docentes, horarios de clase, alumnos por grupo, dificultad de las materias; normas, requisitos de ingreso y requisitos entre materias); dejando ver con esta clasificación que para alcanzar altos niveles de desempeño no es suficiente con las capacidades cognitivas, sino que además se requiere de la vocación y el interés por el estudio. (pp. 64-65)

Como puede notarse, existen diversas clasificaciones de los factores que inciden en el rendimiento académico, algunas de las cuales reconocen en su esencia la complejidad de la medición de dicho constructo, al visualizar una gran variedad de elementos que lo impactan; sin embargo, algunos de ellos hacen referencia a un mismo factor pero denominado de manera diferente. 


\subsection{Factores personales y rendimiento académico}

Una de las conclusiones más relevante que se ha alcanzado en los estudios orientados a explicar el rendimiento académico, es el hecho de que las relaciones entre éste y ciertos aspectos como la edad, el sexo y el nivel socioeconómico se reportan de manera constante, sin importar la técnica de análisis utilizada o el nivel educativo en el que la investigación se realiza (Cervini, 2002). A este respecto, Contreras, Cuevas, Goyenechea e Iturbe (2007) presentan un modelo en el que proponen que el rendimiento académico puede ser estudiado con base en factores como el género, la edad y la escuela de procedencia.

Debido a que los estudios sobre autorregulación reportan que los conocimientos y habilidades de los estudiantes no logran explicar totalmente la variabilidad de dicho constructo, ha sido necesario dar un giro a las investigaciones hacia aspectos motivacionales (Zimmerman, 2008). En este sentido, Salmerón, Gutiérrez-Braojos, Salmeron-Vilchez \& Rodríguez (2011), mencionan que los estudiantes de medicina tienen estrategias de autorregulación más desarrolladas que las de los estudiantes de otras áreas del conocimiento, y que este factor se relaciona con su rendimiento académico, lo que permite suponer que aspectos como la vocación y los intereses juegan un papel importante en el desempeño de los estudiantes de dicha área.

Además, Garello y Rinaudo (2012) comentan que existe una relación directa entre la cantidad de conocimientos que el estudiante adquiere y su motivación, argumentando que ésta se determina por la percepción que el alumno tiene de sus capacidades, así como por las estrategias de enseñanza que el profesor utiliza (tareas que percibe como óptimas, novedosas, con dificultad y relevancia para sus intereses).

También el estudio desarrollado por Libao, Sagun, Tamangan, Pattalitan, Dupa y Bautista (2016) muestra que la motivación tiene una influencia mayor que el sexo, la edad o el grado académico en el rendimiento de los estudiantes. Asimismo, Everaert, Opdecam y Maussen (2017) encontraron que la motivación tiene una influencia positiva en el aprendizaje profundo, lo que conlleva a un rendimiento académico más alto. Estos estudios evidencian la influencia de la motivación en el aprendizaje de los estudiantes de todos los niveles educativos, encontrando que existen momentos en los que la extrínseca tiene un mayor impacto (educación básica) y otros momentos en que la intrínseca determina el aprovechamiento (educación superior).

Por otra parte, existen estudios que encontraron que las expectativas son otro determinante importante del rendimiento académico (Barca, Almeida, Porto, Pe- 
ralbo \& Brenlla, 2012; Caso \& González, 2011; Figuera, Dorio \& Forner, 2003); afirman que los estudiantes con altas expectativas tienden a percibir las situaciones de fracaso como retos y tienen una mejor integración con su entorno educativo, siendo que sus intereses promueven el establecimiento de metas, planes y aspiraciones.

En el mismo sentido, Froiland y Worrell (2017) encontraron en su estudio de las relaciones entre: a) el apoyo a la autonomía parental, b) los objetivos intrínsecos de la vida del estudiante, c) sus expectativas hacia el logro educativo a largo plazo y d) el rendimiento académico; que los objetivos intrínsecos de la vida y las expectativas de los estudiantes se relacionaron moderada y positivamente, y ambos contribuyeron al crecimiento del rendimiento académico.

Otro factor personal que ha sido estudiado es la autoestima. Autores como, Carreras, Fuentes y Tomás (2012), Cruz y Quiñones (2012) y Kususanto, Ismail y Jamil (2010) señalan que existe una relación lineal directa entre el rendimiento académico y la autoestima de los estudiantes, la cual se relaciona con la percepción que estos tienen de sus profesores. En otras palabras, los estudiantes con alta autoestima visualizan que los profesores los apoyan en su proceso de aprendizaje, en tanto que los de baja autoestima los perciben como controladores. En contraparte, estudios como el de Chilca (2017) concluyen que la autoestima no afecta significativamente el rendimiento académico, al menos no en el mismo grado que pueden hacerlo los hábitos de estudio. Resultados como éste evidencian la necesidad de profundizar en el estudio de este tipo de variables, ya que como se ha mencionado, es difícil analizar cada factor sin la influencia de otros.

Otro factor que incide en el rendimiento académico de los estudiantes es el bienestar psicológico; al respecto, Velásquez et al. (2008), mencionan que éste hace que el contexto en el que se desarrollan los procesos formativos sea percibido como propicio para su buen desempeño y que, por el contrario, en su ausencia el mismo contexto puede no ser percibido así. Adicionalmente, Condori (2013) menciona que el bienestar psicológico regula las acciones encaminadas hacia el logro de las metas; es decir, la persona que lo posee es capaz de plantearse metas reales y estar consciente del esfuerzo que implica alcanzarlas.

Como puede verse, los trabajos descritos dan constancia de lo importante de valorar en los estudios y en la puesta en práctica de estrategias que ayuden a los alumnos a mejorar su desempeño, los aspectos no cognitivos que en numerosas ocasiones son más determinantes que otros agentes. Estos elementos pocas veces se analizan y mu- 
cho menos se toman en consideración en los planes de retención de las escuelas; sin embargo, en el presente estudio se consideran como factores determinantes.

Entre los factores personales de tipo cognitivo, autores como Alonso, Táuriz y Choragwicka (2009) y Arquero, Byrne, Flood y González (2009), señalan que el promedio de calificaciones, número de materias reprobadas, tiempo de pasante (en el nivel educativo previo) y la nota de ingreso, son aspectos que influyen en el rendimiento académico. Asimismo, Figuera et al. (2003) señalan que estos factores se vinculan con el proceso de adaptación de los estudiantes a su nuevo entorno educativo, lo cual resulta importante para el buen desempeño académico. De manera específica, Tejedor y García-Valcárcel (2007) concluyen que la variable que más incide en el bajo desempeño de los alumnos son sus conocimientos previos.

Otros estudios como el de Contreras, Gallegos y Meneses (2009) predijeron el rendimiento utilizando una medida de habilidad relativa (ranking), que se obtiene al ordenar a los alumnos según su promedio con relación a sus compañeros de clase; es decir, la posición que ocupa el estudiante con base en su promedio general en su grupo. Cohn, Cohn, Balch y Bradley (2004) incluyeron el ranking como predictor del rendimiento académico en estudiantes de la universidad de Carolina del Sur, encontrando que dicho indicador, aunque resulta significativo, es pequeño en magnitud para predecir el desempeño de los estudiantes. Tal resultado sugiere que utilizar el ranking en combinación con otros indicadores de desempeño, por ejemplo, el promedio de calificaciones, puede producir un indicador del rendimiento académico más eficiente (Martín, 2015).

Por otra parte, Phillips (2011) señala que un factor importante para el buen desempeño académico de los estudiantes de posgrado es la experiencia en investigación. Al respecto, este autor argumenta que todo estudiante de posgrado debe ser usuario inteligente de la producción científica, siendo capaz de valorar la confiabilidad de los resultados de los estudios antes de usarlos, conocer sus limitaciones y saber si estos pueden ser generalizables a otros contextos, además de tener conocimientos de los análisis de datos, entre otras cuestiones.

También se reporta que las estrategias de aprendizaje que caracterizan a los estudiantes impactan el rendimiento académico. Dichas estrategias implican vislumbrar sus habilidades, conocimientos, saber qué deben hacer para aprender, así como advertir las exigencias que tienen las tareas que se les encargan (Vázquez, Noriega \& García, 2013). Un aspecto importante de las estrategias de aprendizaje es que son susceptibles de ser modificadas (Tejedor, 2003), por lo que resulta importante tratar 
de conocer cuáles son las que permiten al estudiante alcanzar altos niveles de desempeño, para promoverlas; además de que estas logran explicar 30\% de la variabilidad del constructo de estudio (Barca et al., 2012).

Desde la perspectiva de los autores de este artículo, el estilo de aprendizaje y las estrategias utilizadas por los estudiantes están determinadas por otros factores de carácter personal como: la disciplina, la capacidad de gestión, la organización del tiempo y la motivación hacia el estudio, por citar algunos; es decir, un estudiante responsable, con buena organización de su tiempo y motivado por la tarea, será poseedor de un conjunto de estrategias de estudio que le permiten alcanzar buenos resultados.

Este hecho sugiere que para realizar un verdadero aporte en la determinación de los factores que influyen en el rendimiento académico, se deben identificar aquellos que no guarden relación con otros que influyan en el desempeño del estudiante, ya que siempre se tendría la duda: ¿son las estrategias de aprendizaje del estudiante las que influyen en el rendimiento académico o es su disciplina, que se relaciona con el uso de buenas estrategias de estudio, la que verdaderamente influye? (Martín, 2015). A este respecto, Montgomery (2004) señala que la interacción de los factores provoca efectos distintos a los reportados al estudiarlos de manera separada, e incluso puede ocultar los efectos individuales. En el mismo sentido, Fernández (2007) menciona que en el estudio de los factores que impactan el rendimiento académico, el análisis de cada uno por separado carece de valor y es el estudio conjunto el que provoca los efectos más importantes sobre dicho constructo.

Como puede observarse, existe un amplio abanico de factores de tipo personal que se reportan como condicionantes del rendimiento académico, lo cual dificulta el estudio de dicho constructo; a lo que se añade el problema de las discrepancias en los resultados de las investigaciones, consecuencia de la diversidad de técnicas de análisis utilizadas, los instrumentos de medición de los factores estudiados y los niveles educativos en los que estas se realizan.

\subsection{Factores institucionales y rendimiento académico}

En cuanto a los factores que atañen a las instituciones educativas, Martín (2015) señala que

los factores institucionales operan a nivel del sistema escolar en su conjunto y a nivel de aula, ya que tienen que ver no únicamente con la enseñanza sino con todas 
las dimensiones del quehacer y la cultura escolar; entre estos, la literatura reporta: la infraestructura, los materiales de enseñanza, el uso del espacio y del tiempo, la organización, las rutinas y las normas, la relación entre directivos y docentes y entre estos, los contenidos de estudio, la pedagogía, la valoración y el uso del lenguaje en las interacciones informales y en la enseñanza, los sistemas de evaluación (premios, castigos, incentivos, estímulos, etc.), por citar algunos. (p. 19)

De manera puntual la literatura reporta que una gestión escolar eficiente tiene un impacto positivo sobre el desempeño escolar de los estudiantes, ya que permite contar con mayores recursos para que el desarrollo de los procesos formativos se dé en mejores condiciones (Paredes \& Paredes, 2009).

Abundando sobre el tema, Heredia (2007) comenta que sobre el rendimiento académico influyen factores que atañen a las instituciones formadoras, que pudieran agruparse en cinco grandes categorías: a) gasto por escuela (recursos destinados a la capacitación de profesores, becas, mantenimiento de infraestructura); b) condiciones materiales de la escuela (acervo bibliográfico pertinente, instalaciones, internet); c) calidad de los maestros (programa de capacitación docente, experiencia docente, nivel de habilitación); d) práctica dentro del salón de clases (planeación de la clase, tiempo del docente en el aula, relación maestro-alumno), y e) administración escolar que ejerce el director (número de alumnos en el colegio y número de alumnos en cada curso).

Por otra parte, Tejedor y García-Valcárcel (2007) comentan que, desde la visión de los profesores, un aspecto que afecta el desempeño de los estudiantes está vinculado con su falta de estrategias para motivarlos; lo cual se debe, entre otras cuestiones, a las múltiples actividades que estos llevan a cabo y que les impiden diseñar tareas que resulten retadoras e interesantes a los estudiantes. Al respecto, Martín (2015) refiere que, en México, los académicos de universidades públicas enfrentan la dificultad de realizar múltiples tareas (actividades de tutoría, gestión, investigación y docencia), limitando el tiempo disponible para la planeación de sus clases y lo que éstas conllevan (preparación de materiales didácticos, organización de las sesiones de clase y actividades motivadoras); además de que la actividad docente está infravalorada en favor de la investigación.

Otro aspecto de impacto se identificó en el estudio realizado por el Laboratorio Latinoamericano de Evaluación de la Calidad de la Educación (LLECE), en el que se encontró que la percepción que tienen los alumnos del clima en el salón de clase afecta su desempeño; siendo que una percepción positiva de éste influye más en los aprendizajes que otros factores relacionados con la institución (Méndez, 2011, como se citó en Martín, 2015). 
En lo que respecta a la importancia que guardan los diferentes tipos de factores en la predicción del rendimiento académico, existen estudios como el de Mella y Ortiz (1999) y Cervini (2002), donde se afirma que aunque existe influencia de los factores institucionales sobre el rendimiento escolar de los estudiantes, dicha influencia es mínima en comparación con la de los factores personales.

\section{Método}

El estudio del cual deriva el presente artículo pertenece al paradigma cuantitativo. Corresponde a una investigación aplicada dado que se orienta a la solución e intervención de situaciones, a través del uso de teorías científicas validadas; es de alcance correlacional y clasifica como un estudio transeccional, puesto que dicha recolección se realizó en un solo momento (Cohen, Manion \& Morrison, 2011).

\subsection{Contexto}

La Facultad de Educación de la Universidad Autónoma de Yucatán, que oferta el programa en estudio, se funda en 1979, pero es hasta 2004 cuando surge la Maestría en Investigación Educativa (MIE). Programa cuyo objetivo es "formar profesionales de la investigación educativa que posean las competencias fundamentales para el diseño y la realización de trabajos de investigación educacional, básica y aplicada, que contribuyan en la identificación, estudio y generación de propuestas de solución a los problemas educativos del entorno" (Universidad Autónoma de Yucatán, 2004); desde su creación, el programa se encuentra en el Padrón Nacional de Programas de Calidad (PNPC) de CONACYT.

\subsection{Participantes}

La población de estudio se conformó de 186 egresados de las cohortes 2004 al 2014. La muestra se determinó a través de un muestreo estratificado; el total de egresados que dio respuesta al instrumento fue de 126; 39 estudiantes de alto rendimiento y 87 de bajo rendimiento; todos ellos becados por CONACYT durante su proceso formativo, por lo que eran estudiantes con dedicación exclusiva al programa. 


\subsection{Instrumento}

Se construyó un cuestionario de respuesta estructurada que indagó sobre los factores personales e institucionales que influyeron en el rendimiento académico de los egresados. Entre los primeros se consideraron: el manejo de las TIC, los conocimientos estadísticos, la comunicación en inglés, el pensamiento crítico, la habilidad de trabajar en equipo, las expectativas, la autoestima, el bienestar psicológico y la motivación al logro (utilizándose para estos tres últimos las escalas de Rosemberg, 1989, de Casullo, 2002 y la de Díaz, Andrade \& La Rosa, 1989, respectivamente). Dicha sección incluyó ocho preguntas abiertas y 119 en escala tipo Likert de cuatro opciones; Totalmente en desacuerdo, En desacuerdo, De acuerdo y Muy de acuerdo. En cuanto a los institucionales se contempló el estudio de los procesos académicos-administrativos (proceso de inscripción y registro de asignaturas, entrega de calificaciones, información sobre el proceso de movilidad, solicitud de examen de grado y normas de impresión de la tesis), el desempeño del asesor y el desempeño del tutor. Esta sección se integró con 25 ítems tipo Likert con las mismas opciones de respuesta que en el apartado de factores personales. Una descripción de los factores considerados en el estudio es la que se presenta en la Tabla 1.

Para la validación del instrumento se realizó un juicio de expertos, solicitándoles realizar una valoración de los ítems de acuerdo con la siguiente escala: Inadecuado y Pertinente. Adicionalmente se pidió realizar un análisis de las instrucciones, aspectos evaluados y opciones de respuesta utilizadas.

Para determinar la validez de constructo se calcularon cuatro medidas descriptivas para cada ítem (media, mediana, desviación estándar y el rango intercuartílico). Para tomar la decisión sobre los ítems que se debían conservar, reformular o suprimir, se establecieron criterios análogos a los utilizados por Barbero, Vila y Suárez (2003), los cuales están en función de las medidas mencionadas: 1) Que el valor de la media de cada ítem fuese igual o superior a 1.5; 2) atender al valor de la mediana, como valor del ítem y, 3) las siguientes consideraciones del rango intercuartílico:

a. Si o $\leq Q_{3}-Q_{1} \leq 0.5$ el ítem se aceptaba.

b. Si $0.5<Q_{3}-Q_{1} \leq 1$ el ítem se revisaba y reformulaba.

c. $S i Q_{3}-Q_{1}>1$ el ítem era rechazado.

En relación con la confiabilidad, ésta se estudió a través del alfa de Cronbach, cuyo valor fue de 0.81 , por lo que el instrumento resulta confiable. 


\section{Tabla 1. Factores en estudio}

\begin{tabular}{|c|c|}
\hline Factor & Descripción \\
\hline Uso de las TIC & $\begin{array}{l}\text { Hace referencia a los conocimientos y habilidades en el manejo de } \\
\text { tecnologías al momento de realizar sus estudios de maestría. }\end{array}$ \\
\hline $\begin{array}{l}\text { Conocimiento } \\
\text { estadístico }\end{array}$ & $\begin{array}{l}\text { Hace referencia a los conocimientos estadísticos de los egresados al } \\
\text { momento de realizar sus estudios de maestría. }\end{array}$ \\
\hline $\begin{array}{l}\text { Comunicación en } \\
\text { inglés }\end{array}$ & $\begin{array}{l}\text { Hace referencia a la habilidad de egresados al momento de realizar sus } \\
\text { estudios de maestría para comprender textos en inglés. }\end{array}$ \\
\hline Pensamiento crítico & $\begin{array}{l}\text { Se refiere a la habilidad del egresado para analizar de manera crítica un } \\
\text { texto del área educativa. }\end{array}$ \\
\hline $\begin{array}{l}\text { Experiencia en } \\
\text { investigación }\end{array}$ & $\begin{array}{l}\text { Busca cuantificar las experiencias adquiridas por los egresados } \\
\text { previamente a su ingreso a la maestría. }\end{array}$ \\
\hline Trabajo en equipo & $\begin{array}{l}\text { Evalúa la habilidad del egresado para realizar trabajos de forma } \\
\text { colaborativa con sus compañeros al momento de estudiar la maestría. }\end{array}$ \\
\hline Expectativas & $\begin{array}{l}\text { Busca medir el nivel de cumplimiento de las expectativas de los } \\
\text { egresados, respecto a diferentes aspectos del programa. }\end{array}$ \\
\hline Autoestima & Mide la valoración que hacen los egresados de sí mismos. \\
\hline Hábitos de estudio & Estrategias utilizadas por el estudiante para favorecer su aprendizaje. \\
\hline Motivación al logro & $\begin{array}{l}\text { Mide la disposición para esforzarse por el éxito en cualquier situación en } \\
\text { la que se aplique un estándar de excelencia. }\end{array}$ \\
\hline Bienestar psicológico & $\begin{array}{l}\text { Esta dimensión evalúa la valoración de los resultados logrados con una } \\
\text { determinada forma de haber vivido para conseguirlos. }\end{array}$ \\
\hline $\begin{array}{l}\text { Procesos académico- } \\
\text { administrativos }\end{array}$ & $\begin{array}{l}\text { Hace referencia a la manera como los diferentes procesos académicos- } \\
\text { administrativos favorecen la obtención del grado. }\end{array}$ \\
\hline Desempeño del asesor & $\begin{array}{l}\text { Hace referencia a la manera como el apoyo y la supervisión del trabajo de } \\
\text { tesis del asesor favorecieron la obtención del grado. }\end{array}$ \\
\hline Desempeño del tutor & $\begin{array}{l}\text { Hace referencia a la manera como el proceso de acompañamiento } \\
\text { académico del tutor favoreció la obtención del grado. }\end{array}$ \\
\hline
\end{tabular}

Fuente: Elaboración propia.

\subsection{Análisis de la información}

El análisis consistió en el estudio de las relaciones entre las variables que aparecen en la Tabla 2 y el rendimiento académico, para lo cual fue necesario construir algunos indicadores en escala de cero a cien para los factores: conocimientos previos (experiencia en investigación, uso de las TIC, conocimientos estadísticos, comunicación en 
inglés, hábitos de estudio, trabajo en equipo y pensamiento crítico), bienestar psicológico, autoestima, motivación al logro, expectativas, procesos académico-administrativos, desempeño del asesor y desempeño del tutor. Dichos indicadores se construyeron en función de las puntuaciones mínimas y máximas de cada una de las respectivas secciones del instrumento, calculándose a partir de la siguiente fórmula:

$$
\text { Indicador }=\left(\frac{\text { Puntuación en la sección }- \text { Puntuación mínima }}{\text { Rango }}\right) \times 100
$$

\section{Tabla 2. Variables consideradas en el estudio}

\begin{tabular}{|c|c|c|}
\hline \multicolumn{3}{|c|}{ Factores personales } \\
\hline 1. Edad & 2. Uso de las TIC & 3. Autoestima \\
\hline 4. Promedio de licenciatura & 5. Conocimiento estadístico & 6. Hábitos de estudio \\
\hline $\begin{array}{l}\text { 7. Número de asignaturas } \\
\text { reprobadas en licenciatura }\end{array}$ & 8. Comunicación en inglés & 9. Pensamiento crítico \\
\hline $\begin{array}{l}\text { 10. Meses transcurridos para } \\
\text { obtener el título de licenciatura } \\
\text { desde el egreso }\end{array}$ & $\begin{array}{l}\text { 11. Bienestar psicológico } \\
\text { - Aceptación-Control } \\
\text { - Autonomía } \\
\text { - Vínculos } \\
\text { - Proyectos }\end{array}$ & $\begin{array}{l}\text { 12. Motivación al logro } \\
\text { - Maestría } \\
\text { - Competitividad } \\
\text { - Trabajo } \\
\text { - Deseabilidad social }\end{array}$ \\
\hline 13. Experiencia en investigación & 14. Trabajo en equipo & 15. Expectativas \\
\hline \multicolumn{3}{|l|}{ 16. Ranking } \\
\hline \multicolumn{3}{|c|}{ Factores institucionales } \\
\hline $\begin{array}{l}\text { 1. Procesos académico- } \\
\text { administrativos }\end{array}$ & 2. Desempeño del asesor & 3. Desempeño del tutor \\
\hline
\end{tabular}

Fuente: Elaboración propia.

Para el caso de los antecedentes académicos (promedio, número de asignaturas reprobadas y meses transcurridos para obtener el título de licenciatura desde el egreso) y la edad, se trabajó con los valores obtenidos directamente del cuestionario. El ranking se definió como el cociente entre la posición que ocupó el estudiante de acuerdo con su promedio en su cohorte de licenciatura y el número de alumnos de dicha cohorte.

Adicionalmente se construyó el indicador de rendimiento académico, entendiendo por éste el cumplimiento de cuatro actividades académicas que denotan la adqui- 
sición de competencias genéricas propias del programa en estudio: 1) Participación en un congreso nacional o internacional con un producto académico derivado de su trabajo de tesis; 2) acreditar al menos una asignatura en una institución de educación superior distinta a la que oferta el programa en estudio; 3) haber realizado una estancia de investigación con algún académico de una institución distinta a la que oferta el programa en estudio por un periodo mínimo de 30 días, y 4) obtener el grado en un periodo máximo de 2.5 años, medidos a partir de la fecha de ingreso al programa, adicionando un punto al indicador por cada actividad realizada.

Una vez construidos dichos indicadores, el estudio de estas relaciones se realizó a través de la Prueba t para muestras independientes (previa verificación del supuesto de normalidad de los datos a través de la prueba de Kolmogórov Smirnov), la Prueba Chi cuadrada de independencia y el coeficiente de correlación de Pearson, dependiendo de la escala de medición de las variables.

Posteriormente, a través de la técnica estadística de Regresión Lineal Múltiple (RLM), por el método Stepwise, se analizó la importancia de cada uno de los factores considerados sobre el rendimiento académico. En la aplicación de la técnica se consideró como variable dependiente al indicador de rendimiento académico y como variables independientes, las que se presentan en la Tabla 2.

Finalmente, con la intención de estudiar el efecto conjunto de los factores que resultaron significativos en el estudio de los factores individuales y en el análisis de la importancia, se aplicó la técnica de Análisis discriminante; clasificándose a los estudiantes en uno de dos grupos: estudiantes de alto rendimiento académico (aquellos que obtuvieron el grado en un periodo menor o igual a 2.5 años, medidos a partir de su ingreso al programa, y que realizaron al menos una de las otras tres actividades descritas en la definición operacional de rendimiento académico) y los estudiantes de bajo rendimiento (aquellos que no cumplieron dichas condiciones); se acordó considerar que un conjunto de variables tendría la capacidad de predecir el rendimiento académico si el porcentaje de buena clasificación de la función discriminante es de al menos $80 \%$.

\subsection{Proceso de recolección de la información}

Con la intención de hacer más ágil la recolección de datos se realizó una versión electrónica del instrumento con el apoyo del servicio Lime Survey, contactándose vía telefónica a los egresados antes de realizar el envío del hipervínculo del instrumento 
por correo electrónico. Cinco días posteriores al envío, se realizaron llamadas telefónicas de seguimiento con el fin de saber quiénes habían dado respuesta, estrategia que continuó hasta cumplir tres meses de recolección. Adicionalmente se contactó a través de Facebook, a aquellos egresados con quienes aún no había podido establecerse comunicación y; con aquellos con los que se estableció contacto pero aún no daban respuesta al cuestionario; se programaron citas para realizar la aplicación personalmente.

\subsection{Aspectos éticos}

Uno de los aspectos éticos considerados en el estudio fue el presentar a los informantes la naturaleza y propósito de la investigación. De igual forma, se respetó el derecho de no participar y de no responder algún ítem del instrumento, así como garantizar el anonimato de la información y el respeto a la dignidad. También se vigiló el cumplimiento cabal de los supuestos requeridos para la aplicación de las técnicas estadísticas utilizadas.

\section{Resultados}

Para estudiar el efecto que de manera individual tienen los diferentes tipos de factores (personales e institucionales) sobre el rendimiento académico, se realizaron los procesos estadísticos que se describen en la Tabla 3, atendiendo a la escala de medición de cada uno de estos.

Del análisis de la Tabla 3 se observa que el manejo efectivo de las tecnologías de la información (uso de las TIC), la experiencia en investigación adquirida a través de la participación en proyectos de investigación o desarrollo de la tesis de licenciatura, los hábitos de estudio, la valoración que hacen los estudiantes de sí mismos (autoestima) y el bienestar psicológico determinan el rendimiento académico.

Para el caso de los factores institucionales, el análisis se realizó a través del coeficiente de correlación de Pearson, correlacionando las puntuaciones de los indicadores de este tipo de variables y las correspondientes al indicador de rendimiento académico, los cuales se presentan en la Tabla 4. 
Tabla 3. Estudio de las relaciones de los factores personales con el rendimiento académico

\begin{tabular}{|c|c|c|c|c|}
\hline Variable estudiada & $\begin{array}{l}\text { Valor del } \\
\text { estadístico } \\
\text { de prueba }\end{array}$ & $\begin{array}{c}\text { Valor de } \\
\text { significancia }\end{array}$ & Técnica & Procedimiento \\
\hline Sexo & 5.400 & 0.065 & \multirow{2}{*}{$\begin{array}{l}\text { Prueba Chi } \\
\text { cuadrada }\end{array}$} & \multirow{2}{*}{$\begin{array}{l}\text { Para el estudio de las relaciones } \\
\text { se categorizó a los estudiantes } \\
\text { en los niveles bajo, medio y } \\
\text { alto, con base en el indicador de } \\
\text { rendimiento académico. También } \\
\text { las licenciaturas se agruparon en } \\
\text { dos categorías: las vinculadas con } \\
\text { el área educativa y las que no. }\end{array}$} \\
\hline Licenciatura de procedencia & 0.180 & 0.913 & & \\
\hline Edad & 0.620 & 0.537 & \multirow{3}{*}{ Prueba t } & \multirow{3}{*}{$\begin{array}{l}\text { Se compararon los valores } \\
\text { de estas variables para los } \\
\text { estudiantes de alto rendimiento } \\
\text { y los de bajo rendimiento. }\end{array}$} \\
\hline Promedio de licenciatura & 0.640 & 0.552 & & \\
\hline $\begin{array}{l}\text { Asignaturas reprobadas en } \\
\text { licenciatura }\end{array}$ & 0.200 & 0.836 & & \\
\hline Ranking & 0.099 & 0.415 & \multirow{13}{*}{$\begin{array}{l}\text { Producto } \\
\text { momento } \\
\text { de Pearson }\end{array}$} & \multirow{13}{*}{$\begin{array}{l}\text { Se exploró la relación entre las } \\
\text { puntuaciones correspondientes } \\
\text { a los indicadores de cada factor } \\
\text { y las correspondientes al valor } \\
\text { del indicador de rendimiento } \\
\text { académico. }\end{array}$} \\
\hline Meses de pasante & 0.290 & 0.130 & & \\
\hline Uso de las TIC & 0.303 & 0.009 & & \\
\hline Conocimientos estadísticos & 0.160 & 0.951 & & \\
\hline Comunicación en inglés & 0.014 & 0.903 & & \\
\hline Pensamiento crítico & 0.107 & 0.367 & & \\
\hline Experiencia en investigación & 0.267 & 0.025 & & \\
\hline Trabajo en equipo & 0.191 & 0.106 & & \\
\hline Hábitos de estudio & 0.448 & 0.001 & & \\
\hline Expectativas hacia el programa & 0.008 & 0.945 & & \\
\hline Autoestima & 0.302 & 0.010 & & \\
\hline Motivación al logro & -0.152 & 0.199 & & \\
\hline Bienestar psicológico & 0.358 & 0.002 & & \\
\hline
\end{tabular}

Fuente: Elaboración propia. 


\section{Tabla 4. Relaciones entre los factores institucionales y el rendimiento académico}

\begin{tabular}{lcc}
\hline \multicolumn{1}{c}{ Factor en estudio } & $\mathbf{r}$ & $\mathbf{p}$ \\
\hline Procesos académico-administrativos & -0.030 & 0.800 \\
\hline Desempeño del asesor & 0.258 & 0.028 \\
\hline Desempeño del tutor & 0.077 & 0.516 \\
\hline
\end{tabular}

Fuente: Elaboración propia.

En la Tabla 4 se evidencia que solamente el desempeño del asesor de tesis es un condicionante del rendimiento académico de los estudiantes. Este hecho deja ver la importancia de considerar la orientación del programa (hacia la investigación) en el análisis del constructo de estudio. De igual forma, revela la importancia de la definición operacional del rendimiento académico, dado que el incluir en ella la condición de obtener el grado, da pie a que el desempeño del asesor haga presente su impacto; lo que pudo no haber ocurrido si se hubiera adoptado una definición más simple de rendimiento académico.

Con la intención de identificar la importancia que sobre el rendimiento académico tiene cada uno de los diferentes factores considerados en el estudio, se aplicó la técnica de regresión lineal múltiple por el método Stepwise, considerando a los coeficientes de regresión estandarizados como el nivel de importancia de estos. Los resultados de dicho análisis se presentan en la Tabla 5.

Como se aprecia en la Tabla 5, los factores que resultan condicionantes importantes son: el promedio de licenciatura, los meses de pasante, la experiencia en investigación, la autoestima, los hábitos de estudio y la motivación al logro, pues estos logran explicar el 56.3\% de la variabilidad del rendimiento académico.

Finalmente, con el interés de identificar la sinergia o efecto conjunto de los factores que resultaron significativos, tanto en el análisis de los efectos individuales como en el estudio de la importancia de estos sobre el rendimiento académico, se aplicó la técnica multivariada de análisis discriminante, clasificando a los estudiantes en uno de dos grupos: estudiantes de alto rendimiento (aquellos que obtuvieron el grado en 2.5 años medidos a partir de su fecha de ingreso al programa y que realizaron alguna de las actividades contempladas en la definición operacional de rendimiento académico) y estudiantes de bajo rendimiento (aquellos que no cumplieron dichas condiciones); los coeficientes de la función discriminante son los que se presentan en la Tabla 6. 


\section{Tabla 5. Importancia de los diferentes tipos de factores sobre el rendimiento académico}

\begin{tabular}{lcclcc}
\hline \multicolumn{1}{c}{ Factor en estudio } & $\begin{array}{c}\text { Coeficientes } \\
\boldsymbol{\beta}\end{array}$ & $\mathbf{p}$ & Factor en estudio & $\begin{array}{c}\text { Coeficientes } \\
\boldsymbol{\beta}\end{array}$ & $\mathbf{p}$ \\
\hline Ranking & -0.185 & 0.472 & Autoestima & 0.260 & 0.028 \\
\hline Edad & 0.102 & 0.359 & Hábitos de estudio & 0.334 & 0.041 \\
\hline $\begin{array}{l}\text { Promedio de } \\
\text { licenciatura }\end{array}$ & -0.249 & 0.024 & Motivación al logro & -0.247 & 0.021 \\
\hline Asignaturas reprobadas & 0.103 & 0.352 & Bienestar psicológico & 0.240 & 0.120 \\
\hline Meses de pasante & -0.249 & 0.021 & $\begin{array}{l}\text { Procesos académico- } \\
\text { administrativos }\end{array}$ & -0.094 & 0.491 \\
\hline Uso de las TIC & 0.119 & 0.480 & Desempeño del asesor & 0.160 & 0.163 \\
\hline $\begin{array}{l}\text { Comunicación en } \\
\text { inglés }\end{array}$ & -0.164 & 0.154 & & & \\
\hline Pensamiento crítico & -0.295 & 0.082 & & & \\
\hline E. en investigación & 0.266 & 0.023 & & & \\
\hline Expectativas & -0.174 & 0.221 & & & \\
\hline Constante & & & $\mathbf{R}^{2}$ & & \\
\hline 2.257 & & 0.563 & & \\
\hline
\end{tabular}

Fuente: Elaboración propia.

Tabla 6. Función discriminante

\begin{tabular}{lc}
\hline \multicolumn{1}{c}{ Factor } & $\begin{array}{c}\text { Función } \\
\text { discriminante }\end{array}$ \\
\hline Autoestima & 0.411 \\
\hline Hábitos de estudio & 0.825 \\
\hline Experiencia en investigación & 0.148 \\
\hline
\end{tabular}

Porcentaje de buena clasificación 80.8

Fuente: Elaboración propia.

Del análisis de la Tabla 6 y de acuerdo con lo establecido en la metodología, el grupo de factores identificados como determinantes importantes tienen también un efecto 
conjunto sobre dicho constructo, logrando predecir en $80.8 \%$ el rendimiento académico de los estudiantes del programa.

\section{Conclusiones}

Una de las primeras conclusiones que se alcanza en el presente estudio se relaciona con el hecho de que no existió un efecto de variables personales como el sexo y la edad sobre el rendimiento académico, contrario a lo reportado por Cervini (2002), quien afirma que el efecto de este tipo de variables se reporta como significativo de manera consistente en los estudios sobre dicho constructo. Esta discrepancia de los resultados podría obedecer a la diferencia de niveles educativos en los que se realizan los estudios.

También llama la atención el hecho de que los conjuntos de factores que se identifican como determinantes del rendimiento académico en el estudio de los efectos individuales y en el análisis de la importancia de estos, no sean los mismos; ejemplo de esto es que aspectos como el promedio de licenciatura, que resultó significativo a través de la técnica de regresión, en concordancia con el trabajo de Alonso, Táuriz y Choragwicka (2009), no resultó así a través de la prueba t, al comparar los promedios de los egresados de ambos grupos (estudiantes de alto rendimiento y bajo rendimiento). Este hecho da idea de la complejidad de la predicción del rendimiento académico y sugiere que dicha discrepancia en los resultados pudiera obedecer, entre otras cuestiones, a las escalas utilizadas para la medición de los factores en estudio. Asimismo, dicha inconsistencia da cuenta de la necesidad de disponer de una medida que evalúe la bondad de los resultados obtenidos a través de cada procedimiento; puesto que si bien ya existen medidas que tienen ese propósito (la potencia de la prueba $[1-\beta]$ para el caso de la prueba t o el coeficiente $\mathrm{R}^{2}$ de bondad de ajuste para el caso de los modelos lineales), éstas no son comparables.

Por otra parte, resulta significativo que aspectos como la licenciatura de procedencia, número de materias reprobadas en licenciatura y el ranking vinculados a los antecedentes académicos, no resulten condicionantes del rendimiento académico, no obstante el haber sido referidos por diferentes autores como determinantes de dicho constructo (Alonso et al., 2009; Arquero et al., 2009; Cohn et al., 2004; Contreras, Gallegos \& Meneses, 2009).

Asimismo, el presente estudio resalta la importancia de los hábitos de estudio como predictores del rendimiento académico, en concordancia con Tejedor 
(2003), quien enfatiza su carácter modificable; es decir, la facilidad con que estos puedan ser mejorados por las instituciones educativas a través de intervenciones curriculares.

De igual forma, el hecho de que la experiencia en investigación resultara consistente como determinante, tal como lo refiere Phillips (2011), hace pensar que la orientación del programa (investigación) es importante en el estudio de los factores que determinan dicho constructo; aunque hay que aclarar que la experiencia en investigación a la que se hace referencia se limita únicamente al desarrollo de tesis de licenciatura o a la participación como becario en algún proyecto de los profesores del programa.

También resalta la incidencia que sobre el rendimiento académico tienen factores como la autoestima, lo cual concuerda con los resultados de Bain et al. (2009), quienes atribuyen a ésta uno de los efectos más importantes sobre el desempeño de los estudiantes.

De todo lo antes expuesto en este apartado, se puede afirmar que dichos factores forman parte de las características del estudiante de alto rendimiento y que son factibles de ser desarrolladas en los estudiantes del programa.

Finalmente, el hecho de que la experiencia en investigación, la autoestima y los hábitos de estudio tuvieran la capacidad de predecir el rendimiento académico de los estudiantes del programa en un $80.8 \%$, deja ver que el análisis conjunto de los factores revela efectos importantes que pudieran ser diferentes a los reportados al estudiar cada factor por separado, pudiendo incluso ocultar los efectos individuales (Fernández, 2007; Montgomery, 2004).

De aquí la importancia de considerar para futuros estudios técnicas multivariadas para el análisis de los factores que se incluyen en este tipo de investigaciones, tales como las pruebas de comparación de Hotelling, Wilks, Roy y Pillai, así como análisis de regresión multivariante y el análisis multivariante de la varianza (MANOva); los cuales pueden ayudar a conocer el efecto conjunto de los factores estudiados de manera más contundente. Asimismo, se debe trabajar en la construcción de instrumentos de medición que arrojen datos en una escala de medición que permita dar cumplimiento a los supuestos para la aplicación de dichas técnicas.

Adicionalmente, los factores analizados en futuros estudios, deben ser determinados en función de los contextos en los que se desarrollan los programas, buscando con esto que el estudio de las relaciones se dé entre grupos de sujetos con características contextuales comunes. 
En lo que respecta a las limitaciones del presente estudio, la más importante hace referencia a los factores que se incluyeron en el instrumento, ya que estos se determinaron a la luz de la revisión de la literatura, dejando de lado las opiniones de los profesores y administradores del programa, así como de los propios alumnos, pudiendo existir otros no considerados en éste.

\section{Lista de referencias}

Albornoz, M. (2004). Particularidades de la institución educativa. Recuperado de https://mayeuticaeducativa.idoneos.com/368115/

Alexander, P. (2006). Psychology in learning and instruction. Nueva Jersey: Pearson.

Alonso, P., Táuriz, G., \& Choragwicka, B. (2009). Valoraciones de Méritos en la Administración Pública y la Empresa: Fiabilidad, Validez y Discriminación de Género. Revista de Psicología del Trabajo y de las Organizaciones, 25(3), 245-258.

Armengol, C., \& Castro, D. (2004). Análisis de los nuevos escenarios universitarios: reflexión previa a los procesos de cambio. Contextos Educativos: Revista de Educación, 6-7, 137-158.

Arquero, J., Byrne, M., Flood, B., \& González, J. M. (2009). Motives, expectations, preparedness and academic performance: a study of students of accounting at a spanish university. Revista de Contabilidad, 12(2), 279-299.

Asociación Nacional de Universidades e Instituciones de Educación Superior. (2017). Anuario estadístico. Población escolar en la Educación Superior. Posgrado, ciclo escolar 2015-2016. Recuperado de http://www.anuies.mx/informacion-y-servicios/informacion-estadistica-de-educacion-superior/anuario-estadistico-deeducacion-superior

Bain, S., Fedynich, L., \& Knight, M. (2009). The successful graduate student: A review of the factors for success. Journal of Academic and Business Ethics. Recuperado de http://www.aabri.com/manuscripts/10569.pdf

Barbero, M. I., Vila, E., \& Suárez, J. C. (2003). Psicometría. Madrid: Universidad.

Barca, A., Almeida, L., Porto, A., Peralbo, M., \& Brenlla, J. (2012). Motivación escolar y rendimiento: impacto de las metas, de estrategias de aprendizaje y autoeficacia. Anales de Psicología, 28(3), 848-859. Recuperado de http://dx.doi. org/10.6018/analesps.28.3.156101

Bronfenbrenner, U. (1987). La ecología del desarrollo humano. Barcelona: Paidós. 
Buchmann, C., \& Diprete, T. A. (2006). The growing female advantage in college completion: The role of family background and academic achievement. American Sociological Review, 71(4), 515-541. Recuperado de http://journals.sagepub.com/doi/abs/10.1177/000312240607100401

Carreras, G., Fuentes, M., \& Tomás, M. (2012). Evaluación de la autoestima de los estudiantes de ESO. Estudio de caso de un IES. Educar, 48(2), 205-227.

Caso, J., \& González, C. (2011). Variables personales, escolares y familiares relacionadas con el rendimiento académico de estudiantes mexicanos de Educación Secundaria. Edupsykhé. Revista de Psicología y Psicopedagogía, 1o(1), 29-48.

Casullo, M. M. (2002). Evaluación del bienestar psicológico en Iberoamérica. Buenos Aires: Paidós.

Cervini, R. (2002). Desigualdades en el logro académico y reproducción cultural en Argentina. Un modelo de tres niveles. Revista Mexicana de Investigación Educativa, 7(16), 445-500.

Chilca, M. (2017). Self-Esteem, Study Habits and Academic Performance among University Students. Propósitos y Representaciones, Revista de Psicología Educativa, 5(1), 101-127. Recuperado de http://dx.doi.org/10.20511/pyr2017.v5n1.145

Cohen, L., Manion, L., \& Morrison, K. (2011). Research Methods in Education ( $7^{\mathrm{a}}$ ed.). Gran Bretaña: Routledge.

Cohn, E., Cohn, S., Balch, D., \& Bradley, J. (2004). Determinants of undergraduate GPAs: SAT scores, high-school GPA and high-school rank. Economics of Education Review, $23(6)$, 577-586.

Condori, L. (2013). Relación de: Autoeficiencia ante el estrés, personalidad, percepción del bienestar psicológico y de salud con el rendimiento académico en universitarios ( Tesis de licenciatura). Universidad Nacional Mayor de San Marcos, Lima, Perú. Recuperada de http://cybertesis.unmsm.edu.pe/handle/cybertesis/3291

Contreras, A., Cuevas, C., Goyenechea, I., \& Iturbe, U. (2007). La sistemática, base del conocimiento de la biodiversidad. México: Universidad Autónoma del Estado de Hidalgo.

Contreras, D., Gallegos, S., \& Meneses, S. (2009). Determinantes de desempeño universitario: ¿importa la habilidad relativa? Revista Calidad en la Educación, 30, 17-48.

Cruz, F., \& Quiñones, A. (2012). Autoestima y rendimiento académico en estudiantes de enfermería de Poza Rica, Veracruz, México. Uni-pluri/versidad, 12(1), 2535. Recuperado de https://aprendeenlinea.udea.edu.co/revistas/index.php/ unip/article/view/13275 
Cruz, S. (julio-octubre, 2006). El rendimiento académico: Desde la práctica de la orientación educativa. Revista Mexicana de Orientación Educativa, 4(9), 41-44. Recuperado de http://remo.ws/revistas/remo-9.pdf

Díaz, F. (1995). La predicción del rendimiento académico en la Universidad: un ejemplo de aplicación de la regresión múltiple. Ediciones Universidad de Salamanca Enseñanza, 13, 43-61. Recuperado de http://revistas.usal.es/index.php/o2125374/article/view/4054

Díaz, L. R., Andrade, P. P., \& La Rosa, L. (1989). Orientación al logro: Desarrollo de una escala multidimensional (EOL) y su relación con aspectos sociales y de personalidad. Revista Mexicana de Psicología, 6(1), 21-26.

Domínguez, D., Sandoval, M., Cruz, F., \& Pulido, A. (2014). Problemas relacionados con la eficiencia terminal desde la perspectiva de estudiantes universitarios. REICE. Revista Iberoamericana sobre Calidad, Eficacia y Cambio en Educación, 12(1), 2534. Recuperado de https://revistas.uam.es/index.php/reice/article/view/2862

Espinosa, E. (2016) Rendimiento académico y eficiencia terminal de los estudiantes de la carrera de fisioterapia del Instituto Politécnico de la Salud, UNAN-Managua, periodo 2013-2015 (Tesis de maestría). Universidad Nacional Autónoma de Nicaragua, Managua. Recuperada de http://repositorio.unan.edu.ni/6067/

Everaert, P., Opdecam, E., \& Maussen, S. (2017). The Relationship between Motivation, Learning Approaches, Academic Performance and Time Spent. Accounting Education, 26(1), 78-107.

Fernández, N. (2007). La educación superior en América Latina. Retos y desafíos hacia el futuro. México: Universidad Autónoma de México.

Figuera, P., Dorio, I., \& Forner, A. (2003). Las competencias académicas previas y el apoyo familiar en la transición a la universidad. Revista de Investigación Educativa, 21(2), 349-369. Recuperado de http://revistas.um.es/rie/article/ view/99251

Froiland, J., \& Worrell, F. (2017). Parental Autonomy Support, Community Feeling and Student Expectations as Contributors to Later Achievement among Adolescents. Educational Psychology, 37(3), 261-271.

Garello, M., \& Rinaudo, M. (2012). Rasgos del contexto para la promoción del desarrollo académico y la creatividad: estudio de diseño con estudiantes universitarios. REICE. Revista Electrónica Iberoamericana sobre Calidad, Eficacia y Cambio en Educación, 10(2), 159-179. Recuperado de https://revistas.uam.es/ index.php/reice/article/view/3062 
Guzmán, M. (2012). Modelos predictivos y explicativos del rendimiento académico universitario: caso de una institución privada en México (Tesis doctoral).Universidad Complutense de Madrid, España. Recuperada de http://eprints.ucm.es/15335/

Heredia, Y. (2007). Factores que afectan el desempeño escolar: el caso de las escuelas primarias públicas de Nuevo León. Memorias del IX Congreso Nacional de Investigación Educativa del Consejo Mexicano de Investigación Educativa, Universidad Autónoma de Yucatán, Mérida, México.

Ibarra, M., \& Michalus, J. (2010). Análisis del rendimiento académico mediante un modelo Logit. Ingeniería Industrial, 9(2), 47-56.

Instituto Nacional de Estadística Geografía e Informática. (2005). Conteo de Población y Vivienda 2005. Recuperado de http://www.beta.inegi.org.mx/proyectos/ccpv/2005/

Kususanto, P., Ismail, H., \& Jamil, H. (2010). La autoestima de los alumnos y su percepción del comportamiento del profesorado: un estudio de agrupación entre clases por capacidades. Electronic Journal of Research in Educational Psychology, 8(2), 707-724. Recuperado de http://dx.doi.org/10.25115/ejrep.v8i21.1395

Libao, N., Sagun, J., Tamangan, E., Pattalitan, A., Dupa, M., \& Bautista, R. (2016). Science Learning Motivation as Correlate of Students' Academic Performances. Journal of Technology and Science Education, 6(3), 209-218.

López-Justicia, D., Hernández, C., Fernández, C., Sánchez, P., \& Chacón, H. (2008). Características formativas y socio afectivas del alumnado de nuevo ingreso en la Universidad. Revista Electrónica de Investigación Psicoeducativa, 6(14), 95-116.

Martín, M. (2015). Optimización del proceso de selección para incrementar la eficiencia terminal en los programas de posgrado (Tesis doctoral). Universidad de Granada, España. Recuperada de https://hera.ugr.es/tesisugr/2569697x.pdf

Mella, O., \& Ortiz, I. (1999). Rendimiento escolar. Influencias diferenciales de factores externos e internos. Revista Latinoamericana de Estudios Educativos, 29(1). Recuperado de http://www.redalyc.org/articulo.oa? id=27029103

Méndez, O. (2011). Calidad de la educación y rendimiento escolar en estudiantes de sexto grado Monterrey, México. Revista de ciencias sociales de la Universidad Iberoamericana, 6(12), 52-78.

Montero, E., Villalobos, J., \& Valverde, A. (2007). Factores institucionales, pedagógicos, psicosociales y sociodemográficos asociados al rendimiento académico en la Universidad de Costa Rica: Un análisis multinivel. RELIEVE, 13(2). Recuperado de https://www.uv.es/RELIEVE/v13n2/RELIEVEv13n2_5.htm 
Montgomery, D. (2004). Diseño y análisis de experimentos (2 $2^{\mathrm{a}}$ ed.). Arizona: Universidad Estatal de Arizona/Limusa Wiley.

Muñoz, J., Martínez, L., \& Armengol, C. (2010). Factores del currículo condicionantes de los resultados escolares. Educar, 46, 87-106. Recuperado de https://doi. org/10.5565/rev/educar.86

Núñez,J., \& González-Pienda, J. (septiembre-diciembre, 2006). Presentación. Papeles del Psicólogo, 27(3), 133-134. Recuperado de http://www.papelesdelpsicologo. es/pdf/1369.pdf

Organización de las Naciones Unidas para la Educación, la Ciencia y la Cultura. (2006). Informe sobre la Educación Superior en América Latina y el Caribe. 200o2005. La Metamorfosis de la Educación Superior. Recuperado de https://www. oei.es/historico/salactsi/informe_educacion_superiorAL2007.pdf

Paredes, R., \& Paredes, V. (2009). Chile: Rendimiento académico y gestión de la educación en un contexto de rigidez laboral. Revista CEPAL, 99, 119-130. Recuperado de https://www.cepal.org/es/publicaciones/37414-revista-cepal-nog9

Phillips, D. (25 de mayo de 2011). ¿Requieren formación en investigación todos los profesionales de alto nivel en educación? Ponencia presentada la Segunda Reunión Internacional de Trabajo. Formación de Investigadores y Profesionales de Alto Nivel en Educación de la Red de Posgrados en Educación, A.C., Zapopan Jalisco, México.

Rivera, N. (2011). Los factores urbanos y el rendimiento académico. México: Universidad Autónoma de Nuevo León.

Rosemberg, M. (1989). Society and the adolescent self-image. Middletown, Nueva York: Weslesyan University Press.

Salmerón, H., Gutiérrez-Braojos, C., Salmeron-Vilchez, P., \& Rodríguez, S. (2011). Metas de logro, estrategias de regulación y rendimiento académico en diferentes estudios universitarios. Revista de Investigación Educativa, 29(2), 467477. Recuperado de http://revistas.um.es/rie/article/view/116041

Tejedor, F. (2003). Poder explicativo de algunas variables determinantes del rendimiento en los estudios universitarios. Revista Española de Pedagogía, 224, 5-32.

Tejedor, F., \& García-Valcárcel, A. (2007). Causas del bajo rendimiento del estudiante universitario (en opinión de profesores y alumnos). Propuestas de mejora en el marco del eees. Revista de Educación, 342, 443-473. Recuperado de http:// www.revistaeducacion.mec.es/re342_21.html 
Torres, E., Osuna, C., \& Liekens, M. (2011). Factores de reprobación en el tronco común de las carreras de medicina y enfermería de la Universidad Autónoma de Baja California, Unidad Ensenada. Trabajo presentado en el XI Congreso Nacional de Investigación Educativa del Consejo Mexicano de Investigación Educativa y la Universidad Autónoma de México, México.

Torres, R., \& Merino, J. (2010). La ejecución real del plan curricular y el uso de los medios $y$ materiales en el mejoramiento del rendimiento académico de los estudiantes de la especialidad de Administración de la Facultad de Ciencias Administrativas y Turismo de la Universidad Nacional de Educación-UNE. (Tesis de licenciatura inédita). Universidad Nacional de Educación Enrique Guzmán y Valle, Perú.

Universidad Autónoma de Yucatán. (2004). Plan de Estudios de la Maestría en Investigación Educativa. Yucatán: Autor.

Vázquez, S. M., Noriega, M., \& García, S. M. (2013). Relaciones entre rendimiento académico, competencia espacial, estilos de aprendizaje y deserción. Revista Electrónica de Investigación Educativa, 15(1). Recuperado de http://redie.uabc. $\underline{\mathrm{mx} / \text { volisno1/contenido-vazqueznoriega.html }}$

Velásquez, C., Montgomery, W., Montero, V., Pomalaya, R., Dioses, A., Velásquez, N., Araki, R., \& Reynoso, D. (2008). Bienestar psicológico, asertividad y rendimiento académico en estudiantes universitarios sanmarquinos. Revista de Investigación en Psicología, 11(2), 139-152. Recuperado de http://dx.doi. org/10.15381/rinvp.v11i2.3845

Zimmerman, B. J. (2008). Investigating self-regulation and motivation: Historical, background, methodological developments, and future prospects. American Educational Research Journal, 45(1), 166-183. Recuperado de http://journals. sagepub.com/doi/abs/10.3102/0002831207312909 